The present experiments provide strong evidence that $\beta$-asarone is bioactivated via microsomal oxidation to a bioactive epoxide. Synergism of $\beta$-asarone by menthol represents the first reported case of synergism between two natural products where the synergism results from metabolic bioactivation by enzymes usually involved in detoxication.

Acknowledgments. We thank Nancy Brard and Pathmini Maheswaran for technical assistance and Cathy McCloskey and Tom Lowery for their comments on the manuscript. Supported by grants from the Natural Sciences and Engineering Research Council of Canada to M. B. Isman.

* Current address: Agriculture Canada Research Station, Summerland, $\mathrm{BC}$, Canada VOH $1 \mathrm{ZO}$.

** Author for correspondence and reprint requests.

1 Saxena, B. P., Koul, O., Tikku, K., and Atal, C. K., Nature, London 270 (1977) 512.

2 Koul, O., Tikku, K., and Saxena, B. P., Experientia 33 (1977) 29.

3 Koul, O., Smirle, M. J., and Isman, M. B., J. chem. Ecol. 16 (1990) 1911.
4 Koul, O., in: Sci. Acad. Medal Lectures, p. 62. Ed. INSA. New Delhi, India 1979.

5 Matolcsy, G., Farag, A. I., Varjas, L., Belai, I., and Darwish, Y. M., in: Juvenile Hormone Biochemistry, pp. 393-402. Eds G. E. Pratt and G. T. Brooks. North-Holland Biomedical Press 1981.

6 Muller, P. J., Masner, P., Kalin, M., and Bowers, W. S., Experientia 35 (1979) 704.

7 Pratt, G. E., Jennings, R. C., Hamnett, A. F., and Brooks, G. T., Nature, London 284 (1980) 320.

8 Moldenke, A. F., Berry, R. E., and Terriere, L. C., Comp. Biochem. Physiol. 74C (1983) 365.

9 Mattocks, A. R., and White, I. N., Chem. Biol. Interactions 3 (1971) 383.

10 Boyd, M. R. Nature, London 269 (1977) 713.

11 Jollow, D. J., Mitchell, J. R., Zampaglione, N., and Gillette, I. R., Pharmacology 11 (1974) 151.

12 Rotman, A., Life Sci. 21 (1977) 891.

13 Soderlund, D. M., Messeguer, A., and Bowers, W. S., J. Agric. Food Chem. 28 (1980) 724.

$0014-4754 / 90 / 101082-03 \$ 1.50+0.20 / 0$

(C) Birkhäuser Verlag Basel, 1990

\title{
A novel reaction time task for investigating force and time parameters of locomotor initiation in rats
}

\author{
W. Hauber \\ Dept. Neuropharmacology, University of Tuebingen, Mohlstr. 54/1, D-7400 Tuebingen (Federal Republic of Germany) \\ Received 20 February 1990; accepted 15 May 1990
}

Summary. A novel simple reaction-time task for rats is described in the present study. Food-deprived rats were trained in a modified runway for rapid locomotor initiation, in response to a combined optical/acoustic stimulus, to receive a food reward. Rats rapidly learned this task with small variability, and movement patterns of locomotor initiation are congruent under these conditions. Reaction time, movement time and accelerative forces were recorded from each initiation of locomotion by means of video equipment and a force platform. The quantification modes yielded consistent results and a quantitative description of measured force and time parameters is given. The task may be especially appropriate for investigating basal ganglia functions. The present results will be the basis for investigating initiation of locomotion in animal models of neurological diseases.

Key words. Locomotor initiation; reaction time; movement time; force; basal ganglia; rat.

Since several aspects of movement initiation are disturbed in neurodegenerative disorders of the basal ganglia, such as Parkinson's disease (Marsden ${ }^{1}$ and references cited) or Huntington's disease ${ }^{2,3}$, and during normal aging ${ }^{4}$, the study of voluntary movement initiation has been an issue of intense interest. A dysfunction of the nigrostriatal dopamine (DA) system has been implicated in these movement-initiation deficits and in bradykinesia of humans ${ }^{1}$. Animal studies using movement initiation as a paradigm for investigating basal ganglia functions provided further evidence that an intact nigrostriatal DA system appears to be necessary for rapid response to an external stimulus ${ }^{5}$.

Different types of apparatus have been designed to study movement initiation in rats. Reaction time (RT) performance has mostly been assessed in lever-release tasks conducted in operant chambers. It has been shown that RT performance depends on a number of variables in- cluding integrity of the nigrostriatal DA system ${ }^{6,7}$. However, most rodent models using RT tasks have focused on distal paw movements, disregarding the involvement of the basal ganglia in the control of axial and proximal movements ${ }^{8,9}$. The relationship between basal ganglia and axial motor control is indicated by anatomical and electrophysiological data ${ }^{10-12}$. In addition, clinical findings with regard to Parkinson's disease have revealed severe deficits in control of posture and gait, and initiation of proximal movements ${ }^{13}$.

With respect to locomotion, it was suggested that a lack of regulation by descending basal ganglia efferents is reflected in a deficient initiation of locomotion ${ }^{8}$, while the stepping mechanism as such is relatively unaffect$\mathrm{ed}^{14}$. Rodent studies paralleled these findings in part, showing a disturbed initiation of locomotion using different models ${ }^{15-17}$. These studies, however, were not performed under RT conditions with relatively high ki- 
netic requirements for the response, which is known to be especially sensitive to basal ganglia dysfunction ${ }^{18}$.

The work presented here describes a new, simple reaction-time task for rats. The animals were trained for rapid initiation of locomotion in response to an external stimulus to receive a food reward. The device permitted a detailed analysis of the process of locomotor initiation in terms of reaction time (RT) and movement time (MT). In addition, the forces emitted by an animal during movement initiation were recorded by a force platform, which provided dynamic index of the proximal movement required in this condition.

\section{Materials and methods}

Subjects. Subjects were nine male (weight range $230-$ $250 \mathrm{~g}$ ) Sprague Dawley rats (Interfauna, Tuttlingen, FRG) caged in two groups with free access to water. They were provided with $12 \mathrm{~g} / \mathrm{d} /$ rat rodent laboratory chow after the daily experimental sessions. The colony room was temperature-regulated $\left(22 \pm 3{ }^{\circ} \mathrm{C}\right)$ and rats were maintained on a $12-12 \mathrm{~h}$ light dark cycle (lights on at $06.00 \mathrm{~h}$ ).

Apparatus. The apparatus was built of plexiglass and consisted of a removable start and goal box $(22 \times 9 \times 9 \mathrm{~cm}$ respectively) which were connected by a runway $(100 \times 9 \times 9 \mathrm{~cm})$ (see fig. 1).

A movable front door blocked the runway entrance. The door was installed in a frame and operated electromagnetically. Once opened, the door dropped vertically downwards (duration $<80 \mathrm{~ms}$ ). In front of the start box, lamps were mounted bilaterally and a loudspeaker was attached above. A combined optical-acoustic $(10 \mathrm{~W}, 8 \mathrm{kHz} / 40 \mathrm{~dB})$ stimulus signalled the simultaneous opening of the front door. Perpendicular to the start box, a video camera (Panasonic WV 130 CCD) was installed in the plane of the runway, connected with a time generator (Panasonic WJ 810) (clock pulse rate: 10-ms intervals) and a recorder (Panasonic AG 6200).

A force-measuring unit was mounted below the start box to monitor the anterior-posterior (horizontal) and verti-

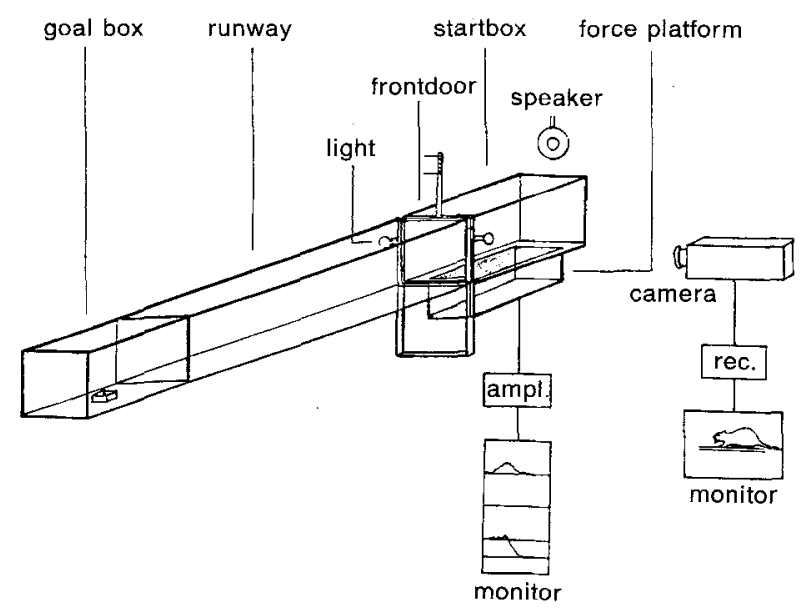

Figure 1. Schematic representation of the apparatus. cal components of the resulting force applied by an animal on the surface. The mediolateral (horizontal) force component was disregarded. The force platform was newly designed and constructed, since suitable products are commercially not available. Furthermore, some designs described in the literature ${ }^{19}$ do not easily lend themselves to miniaturization or reconstruction. The force platform was built from force sensors used for construction of precision topload balances. The force sensor (Soehnle, Murrhardt, FRG) consisted of a strain gauge as a force-sensing element, which was installed in a frame. The frame served as a force-transmitting device, and was compensated for decentral force application. In addition, temperature and humidity compensation was provided by appropriate construction and use of materials (according to the manufacturer's declaration).

Two force sensors were suitably assembled to allow separate recording of the force components. The assembled force sensors were connected with a top plate serving as the animal's support in the start box, attached to a vibration-absorbing, adjustable base frame. Each full bridge circuit of strain gauges was connected with an operation amplifier, digital memory oscilloscope (ITT-metrix OX $750 / 2$ ), and printer (Laumann, Servocass 2000). The first prototype of the design was tested in pilot studies and then improved in some minor aspects. The final version has been used for several months and has provided reliable and precise force measurements (table).

Behavioral procedure. Naive, untreated rats were habituated individually for $10 \mathrm{~min}$ to the baited apparatus prior to the onset of training. The following day, animals were trained in one session per day, consisting of 10 consecutive trials per animal. A food-deprived rat was placed in the start box facing the closed front door blocking the entrance to the runway and goal box with the food reward (pellet, Noyes, $45 \mathrm{mg}$ ). After a variable delay (3$10 \mathrm{~s}$ ), the stimulus signalled the simultaneous opening of the front door. The rats were trained to initiate locomotion in response to the stimulus and to move straight ahead through the runway into the goal box for the reward in a baited cup. Approximately $10 \mathrm{~s}$ later the rat was again placed in the start box for a new trial.

Evaluation. The transition from stance to gait of each locomotor initiation was recorded by means of video equipment and the force platform. RT was monitored in all training sessions, force and movement time (MT) data were recorded from the last session. Only correct reac-

Force platform - technical data

\begin{tabular}{lrr}
\hline Calibrated range & $\mathrm{Fy}, \mathrm{Fz}$ & $0.2-20 \mathrm{~N}$ \\
Threshold & $\mathrm{Fy}, \mathrm{Fz}$ & $0.2 \mathrm{~N}$ \\
Linearity & $\mathrm{Fy}, \mathrm{Fz}$ & $<1.5 \%$ \\
Hysteresis & $\mathrm{Fy}, \mathrm{Fz}$ & $<0.5 \%$ \\
Sensitivity & $\mathrm{Fy}, \mathrm{Fz}$ & $0.1 \mathrm{~N}$ \\
Crosstalk & $\mathrm{Fy}-\mathrm{Fz}$ & $<1 \%$ \\
& $\mathrm{Fz}-\mathrm{Fy}$ & $<1 \%$ \\
Natural frequency & $67 \mathrm{~Hz}$ \\
Deviation due to decentral force application on top plate & $<1 \%$ \\
\hline
\end{tabular}


tions (initiation of locomotion within $100-1000 \mathrm{~ms}$ ) were considered for evaluation. RT data were collected by frame-by-frame analysis from the video records and $\mathrm{RT}$ was defined as latency from stimulus presentation up to the lifting of a paw from the surface. MT was determined as the duration from the lifting of the first paw until the time when the last paw left the top plate. Forcetime waveforms of each response were detected by means of the force platform, and recorded on-line. The measurements and calculations of force parameters were performed after the data had been collected. The horizontal component of each waveform was manually analyzed, including 2 parameters: the maximum (peak) force defined as the distance between baseline and maximum force value, and the rate of force development defined as the slope from the initial force increase to the peak force value.

\section{Results}

All animals rapidly learned the simple reaction-time task presented here with little variability, and quantification of the analyzed parameters yielded consistent results. The movement patterns of locomotor initiation under these conditions were congruent and were established within a few trials: during the prestimulus phase a rat remained with all 4 paws on the ground in an upright posture to initiate locomotion. Following stimulus presentation, locomotion was usually initiated by a forepaw ( $>95 \%$, $\mathrm{n}=374$ ), and a rat rapidly moved to the goal box. Initially observed impairments of movement initiation, e.g. inadequate postures or paw positions, were disregarded for evaluation. Paw preferences were not investigated, though they presumably occur. As illustrated in figure 2, the rats showed a steady increase of correct trials in consecutive sessions, reaching $97 \%$ correct trials on day 6.

Mean correct RT data exhibited that baseline performance (range 238.3-248.7 ms) was already established

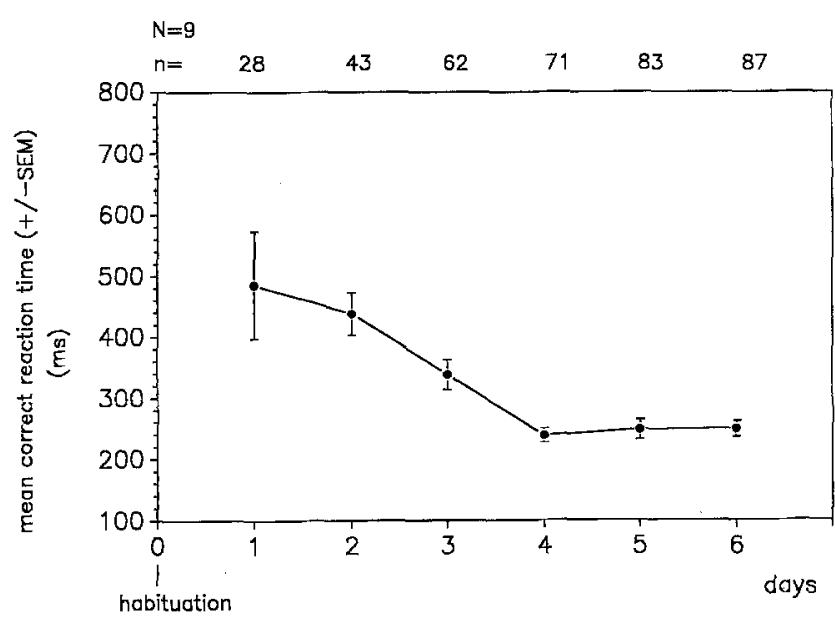

Figure 2. Mean correct reaction times $( \pm$ SEM) and number of correct responses (n) measured in consecutive daily sessions consisting of 10 trials per animal $(\mathrm{N}=9)$, respectively. On day 0 the animals were habituated individually to the apparatus.
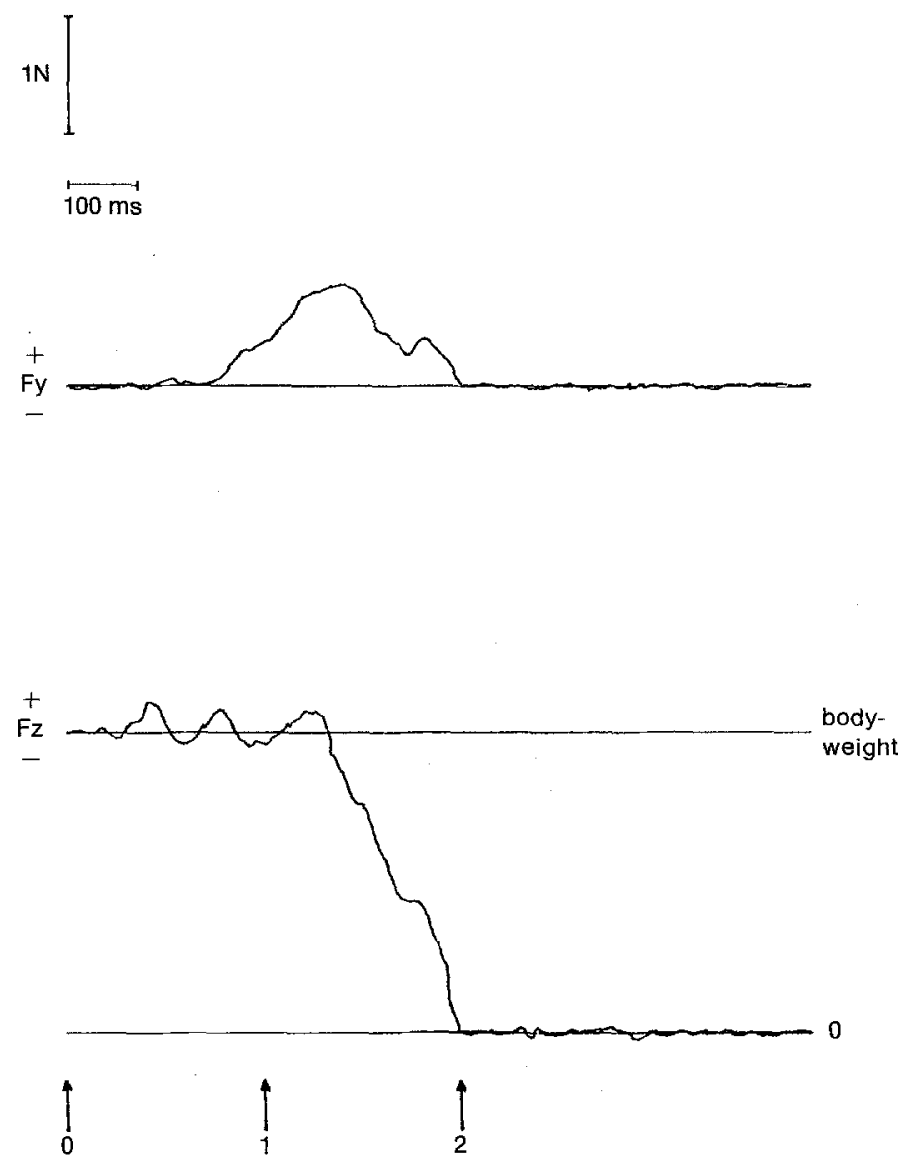

Figure 3. Representative force time waveform from a locomotor initiation. Numbers on time axis mean $0=$ stimulus presentation, $1=\mathrm{RT}$ (from video signal), 2 = leaving the platform. Top: horizontal anteriorposterior force component (+Fy: forward, - Fy backward) of a rat weighing $234 \mathrm{~g}$. Bottom: vertical force component $(+\mathrm{Fz}$ : force increase, - Fz: force decrease)

on day 4 and remained nearly constant. In addition, variability of RT decreased, as indicated by the narrowing of the standard errors of the means. Measures of MT showed a mean duration of $608.9 \mathrm{~ms}(\mathrm{SEM} \pm 38.1 \mathrm{~ms}$, $n=64$ ), indicating relatively uniform movement patterns. An impact of the prestimulus position of a rat on MT data could be neglected, as the start box dimensions confined the animals to a relatively defined position, and the rats spontaneously occupied a position close to the front door. Analysis of force-time patterns of ground reaction forces yielded different results with regard to quantification (fig. 3).

The vertical force-time peaks, reflecting movements in that plane, exhibited inconsistent and irregular patterns which made quantitative analysis difficult. This may be due to differences in postural preparation of locomotor initiation. To address this question, single paw recordings are required for a more detailed analysis of postural preparation of locomotor initiation. These data were therefore excluded from further analysis. In contrast, the anterior-posterior (horizontal) force component, reflecting the animal's propulsion, showed reproducible pat- 
terns which were easy to analyze. Two parameters known to be drug-sensitive ${ }^{20}$ were used to characterize this peak: the rate of force development (slope) and the maximum (peak) force. The average rate of force development was found to be $8.7 \mathrm{~N} / \mathrm{s}(\mathrm{SEM} \pm 0.6, \mathrm{n}=73)$ and the mean peak force $0.6 \mathrm{~N}$ (SEM $\pm 0.025, \mathrm{n}=73$ ). Comparison of peak force timing and corresponding $\mathrm{RT}$ revealed that the peak force value was achieved simultaneously or immediately after movement initiation ( $>94 \%, \mathrm{n}=73$ ). The quantitative description of initiation of locomotion given here was confirmed under the conditions of a study investigating drug effects using the same system ${ }^{20}$.

\section{Discussion}

The results indicate that initiation of locomotion in rats is a consistent response which is reliably quantifiable in the simple reaction-time task presented here. Gradual food deprivation was sufficient to produce consistent responses. On the whole, the animals rapidly learned this task and a constant performance was established in a few trials. This holds true both for initiation of locomotion and for completion of the task. Once trained, rats moved straight forward without interruption to the goal box to receive the food reward. The appetitive nature of the task used here is a major advantage, since most rodent RT tasks are conducted using aversive patterns, such as active avoidance with shock treatment, which may confound performance in various ways.

The RT data collected here are difficult to compare with other RT studies as this is, to our knowledge, the first study investigating locomotor initiation under R $\mathrm{T}$ conditions. However, lever-release tasks have revealed RT in a range of about $200-400 \mathrm{~ms}$ and under specific circumstances less than $200 \mathrm{~ms}^{6,7,21}$, and the maximum reactive capacity of Sprague-Dawley rats was estimated to be approximately $120 \mathrm{~ms}^{22}$. These findings are in line with our RT results. Therefore, it may be concluded that initiation of a proximal movement as examined here is a relatively fast response initiated with latencies similar to those for manipulatory movements. In addition, latencies of locomotor initiation indicate a voluntary response initiation, as involuntary reflexive movements (e.g. startle, jump) have shorter latencies (less then $120 \mathrm{~ms})^{22}$. However, these comparisons are limited for methodological reasons. There are a number of factors (age, body weight, spontaneous activity levels, individual speed preference) which potentially interact with response performance. However, these primarily affect response completion and not movement initiation ${ }^{21}$. Furthermore, the narrow age and weight range of rats used here and the consistency and small variability of the results indicate that these variables were not important.

The force measurements in this study were focused on the force-time waveforms of the horizontal force component representing the propulsive force generating the initial acceleration during locomotor initiation. Regarding the timing of propulsive force production and movement initiation, the data indicated that the onset of force production preceded movement initiation, and the peak force was achieved with or immediately after movement initiation. Thus, force and time parameters appear to be conveniently coordinated during locomotor initiation. Both parameters used to quantify this waveform yielded consistent results and may be an index of the dynamic characteristic of this proximal movement. As dynamic abilities of rats are rarely investigated, the results presented here are difficult to classify, but available data concerning peak forces ${ }^{23-25}$ resemble those measured in this study. Furthermore, subtle chances of force production seem to be associated with a dopaminergic dysfunction in rats and humans ${ }^{24-26}$.

Taken together, the novel method presented here permits a quantitative description of force and time parameters of rapid locomotor initiation in rats. It is known that these parameters may be altered as a result of basal ganglia dysfunctions (see introduction). The demand of a fast axial body movement in this task may be especially appropriate for investigating basal ganglia functions, and this test closely parallels a corresponding task in man which is sensitive to slowed movement-initiation in Parkinson's disease (Diener, personal communication). In addition, measurement of axial motor control was recently proposed to be a useful tool in evaluating therapeutic drugs in Parkinson's disease ${ }^{27}$.

On the basis of the present analysis, it will be possible to discern the effects of impaired basal ganglia functions in suitable rodent models and then to compare the effectiveness of drug treatments. Preliminary studies using the task presented here showed that the force and time parameters are sensitive to subtle changes of central dopaminergic transmission $20,28,29$.

Acknowledgments. I wish to thank Dr Peter Lindenmüller, University of Stuttgart (Institute of Design and Production in Precision Engineering) for developing and constructing the force measuring unit and Prof Dr Werner J. Schmidt who encouraged this study. I am also grateful to Paul Schmidt for improving the English of an earlier version of the paper. The research was supported by the Deutsche Forschungsgemeinschaft (SFB 307).

1 Marsden, C. D., Movement Disorders 4 Suppl. 1 (1989) S26-S37.

2 Hefter, H., Hömberg, V., Lange, W. W., and Freund, H. J., Brain 110 (1987) 585.

3 Thompson, P. D., Berardelli, A., Rothwell, J. C., Day, B. L., Dick, J. P. R., Benecke, R., and Marsden, C. D., Brain 111 (1988) 223.

4 Birrin, J. E., Woods, A. M., and Williams, M. V., in: Brain Function in Old Age, pp. 10-14. Eds F. Hoffmeister and C. Mueller. Springer, Berlin 1979.

5 Hassler, R., J. neurol. Sci. 36 (1978) 187.

6 Amalric, M., and Koob, G. F., J. Neurosci. 7(7) (1987) 2129

7 MacRae, P. G., Spirduso, W. W., and Wilcox, R. E., Brain Res. 451 (1988) 139.

8 Garcia-Rill, E., Brain Res. Rev. 11 (1986) 47.

9 Labuszewski, T., Lockwood, R., McManus, F. E., Edelstein, L. E., and Lidsky, T. I., Exp. Neurol. 74 (1981) 93

10 Garcia-Rill, E. A., Nieto, A., Adinolfi, C. D., and Buchwald, N. A., Brain Res. 170 (1979) 393.

11 Anderson, M. E., Brain Res. 143 (1977) 325.

12 DeLong, M. R., J. Neurophysiol. 34 (1971) 414. 
13 Birkmayer, W., and Riederer, P., Parkinson's disease. Springer, Wien 1986.

14 Martin, J. P., in: The Basal Ganglia and Posture, pp. 20-35. Pitman, London 1967.

15 Posluns, D., Psychopharmacologia 3 (1962) 361.

16 Price, M. T. C., and Fibiger, H. C., Pharmacol. Biochem. Behav, 3 (1975) 285.

17 Dunnett, S. B., and Björklund, A., Neurosci. Lett. 41 (1983) 173.

18 Spirduso, W. W., Gilliam, P. E., Schallert, T., Upchurch, M., Vaughn, D. M., and Wilcox, R. E., Brain Res. 335 (1985) 45.

19 Lywood, D. W., Adams, D. J., van Eyken, A., and Macpherson, J. M., Med. Biol. Eng. Comput. 25 (1987) 698.

20 Hauber, W., and Schmidt, W. J., Behav. Brain Res. (1990) in press.

21 Spirduso, W. W., Abraham, L. D., and Wolf, M. D., Pharmac. Biochem. Behav. 14 (1981) 433

22 Spirduso, W. W., and Farrar, R. P., J. Geront. 36 (1981) 654.

23 Falk, J. L., Pharmac. Behav. 4 (1969) 421.
24 Fowler, S. C., LaCerra, M. M., and Ettenberg, A., Pharmac. Biochem. Behav. 25 (1986) 791.

25 Ettenberg, A., Neurosci. Biobehav. Rev. 13 (1989) 105.

26 Stelmach, G. E., and Worringham, C. J., Neuropsychologia 26 (1988) 93.

27 Weinrich, W. M., Koch, K., Garcia, F., and Angel, R. W., Neurology 38 (1988) 540.

28 Hauber, W., in: Contributions to the 17th Göttingen Neurobiology Conference, p. 357 . Eds N. Elsner and W. Singer. Thieme, Stuttgart 1989.

29 Hauber, W., in: Contributions to the 18th Göttingen Neurobiology Conference, p. 448 . Eds N. Elsner and G. Roth. Thieme, Stuttgart 1990.

$0014-4754 / 90 / 101084-05 \$ 1.50+0.20 / 0$

(C) Birkhäuser Verlag Basel, 1990 